\title{
Low-Dose Radiation Therapy for Primary Conjunctival Marginal Zone B-Cell Lymphoma
}

\section{Ga-In Lee, MD ${ }^{1}$ \\ Dongryul Oh, MD, PhD² \\ Won Seog Kim, MD, PhD 3 \\ Seok Jin Kim, MD, PhD ${ }^{3}$ \\ Young Hyeh Ko, MD, PhD ${ }^{4}$ \\ Kyung In Woo, MD, PhD' \\ Yoon-Duck Kim, MD, PhD' \\ Yong Chan Ahn, MD, PhD²}

Departments of ${ }^{1}$ Ophthalmology and ${ }^{2}$ Radiation Oncology, ${ }^{3}$ Division of Hematology and Oncology, Department of Internal Medicine, ${ }^{4}$ Department of Pathology, Samsung Medical Center, Sungkyunkwan University School of Medicine, Seoul, Korea
Correspondence: Kyung In Woo, MD, PhD Department of Ophthalmology,

Samsung Medical Center, Sungkyunkwan University School of Medicine, 81 Irwon-ro, Gangnam-gu, Seoul 06351, Korea

Tel: 82-2-3410-3570

Fax: 82-2-3410-0074

E-mail: eyeminded@skku.edu

Co-correspondence: Yong Chan Ahn, MD, PhD Department of Radiation Oncology,

Samsung Medical Center, Sungkyunkwan University School of Medicine, 81 Irwon-ro, Gangnam-gu, Seoul 06351, Korea

Tel: 82-2-3410-2612

Fax: 82-2-6190-5332

E-mail: ahnyc@skku.edu

Received April 14, 2017

Accepted June 5, 2017

Published Online June 16, 2017

${ }^{*}$ Ga-In Lee and Dongryul Oh contributed equally to this work.

\section{Purpose}

The purpose of this study was to evaluate the clinical features and the long-term outcomes of primary conjunctival marginal zone B-cell lymphoma (MZBCL) patients who were treated with radiation therapy $(\mathrm{RT})$.

\section{Materials and Methods}

Retrospective data of 79 patients with 121 primary conjunctival MZBCL lesions were collected from January 1, 2001 till June 30, 2014. All lesions were treated by local RT (26 Gy) with patient-specific customized lens-shielding device.

\section{Results}

The current Korean patients' cohort showed younger median age at diagnosis (38 years), great female preponderance (78.5\%) and more frequent bilateral involvement (53.2\%) than the previous studies. Following 26 Gy's RT, excellent clinical outcomes were achieved: 5-year rates of overall survival, local relapse-free survival, and contralateral relapse-free survival were $100 \%, 98.1 \%$, and $91.5 \%$, respectively. Two patients $(2.5 \%)$ developed local relapse and five $(6.3 \%)$ developed relapse at initially uninvolved contralateral conjunctiva with median interval of 52.9 months, and late adverse events of grade 2 and 3 occurred in seven (8.8\%) and two (2.5\%) patients, respectively.

\section{Conclusion}

26 Gy's RT was highly effective and safe, with the use of lens-shielding device, in treating patients with primary conjunctival MZBCL.

\section{Key words}

Conjunctiva, Marginal zone B-cell lymphoma, Radiotherapy 


\section{Introduction}

Lymphomas arising in the conjunctiva include a heterogeneous group of histologic subtypes. Systematic review including 1,014 conjunctival lymphoma patients from several Western countries showed that B-cell lineage accounted for a vast majority $(98 \%)$ and the most common subtype was extranodal marginal zone B-cell lymphoma (MZBCL) of mucosa-associated lymphoid tissue, which is synonymous with mucosa-associated lymphoid tissue lymphoma $(81 \%)$ [1]. There have been various options in treating conjunctival MZBCL ranging from observation alone, single modality of local or systemic therapy, and to combinations of local and systemic modalities [1,2]. Though either observation alone following biopsy was suggested by some groups based on spontaneous regression $[3,4]$, or antimicrobial doxycycline was tried to those with active chlamydial infection $[5,6]$, local radiation therapy (RT) has traditionally been recommended based on high local progression rate following these approaches [7-13].

There are many clinical reports on RT in treating primary adnexal ocular lymphoma; however, the reports which exclusively concentrated on primary conjunctival lymphoma are scarce $[7,8,12,14,15]$. Conjunctival lymphoma has distinctive clinical features compared with orbital lymphomas involving other subsites. It is relatively easy to evaluate the primary lesion without expensive imaging modalities. In addition, it has relatively small tumor volume but is closer to the corneal epithelium and the lens. Thus, low to moderate dose of RT (20-30 Gy) have been used in the literature $[2,10,14,16]$. However, there is no clear evidence regarding RT dose as yet.
The current study aimed to evaluate the clinical features and the long-term outcomes focused on conjunctival MZBCL patients who were treated with 26 Gy of RT. To the best of our knowledge, this is the largest patient series of conjunctival MZBCL treated with single modality of RT in a single center.

\section{Materials and Methods}

\section{Patients}

Primary conjunctival lymphoma is defined as the main lesion originating from the conjunctival fornix and/or the bulbar conjunctiva, but not the extension of the lesion to the subconjunctival space from other subsites of the orbit. Authors retrieved 95 patients who were pathologically diagnosed as having primary conjunctival lymphoma at our institution from January 1, 2001, to June 30, 2014. The current study was performed in accordance with the principles of the Declaration of Helsinki, and the institutional review board and ethics committee at our institution approved the study protocol.

All patients underwent the initial staging workups including thorough physical examination, complete blood count (CBC), liver function test, lactate dehydrogenase (LDH), $\beta 2$ microglobulin level, chest X-ray, computed tomography (CT) scans of the orbit, chest, abdomen, and pelvis, and bone marrow aspiration and biopsy from the iliac bone.

According to World Health Organization classification, MZBCL was the most common in 90 patients $(94.7 \%)$ and

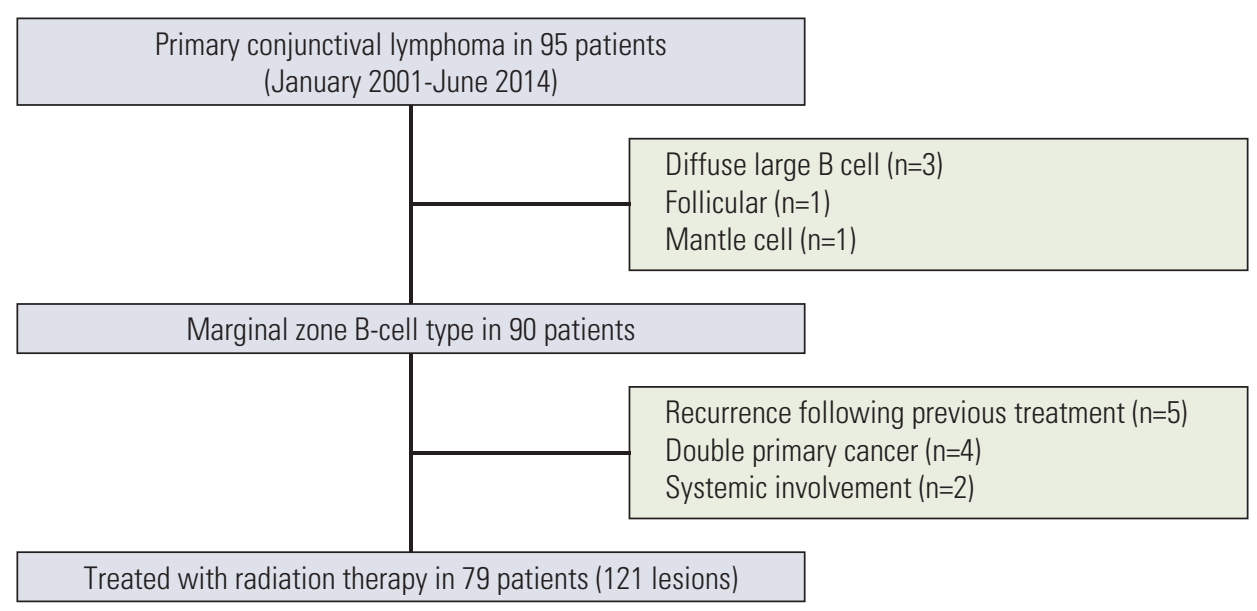

Fig. 1. Patients' enrollment. 

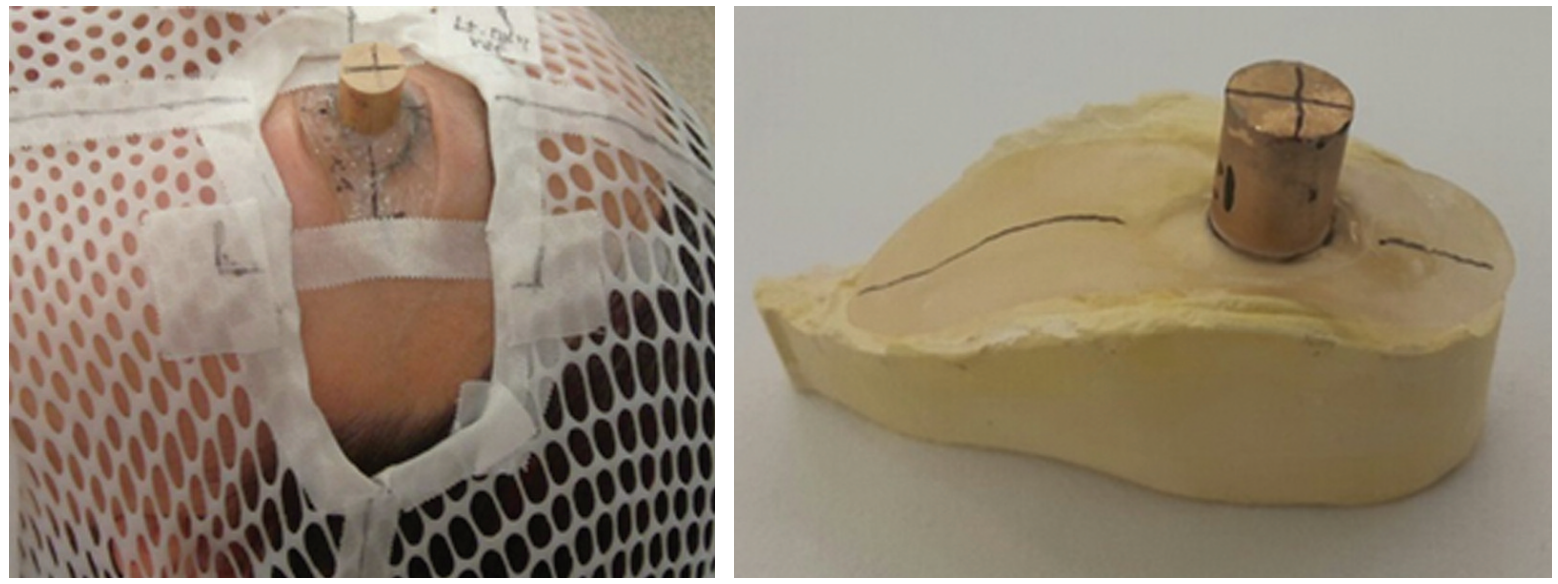

Fig. 2. The customized lens-shielding block, composed of a cerrobend alloy column mounted on the individually custommade acryl contact lens, was used to avoid irradiation to the lens.

other histologic subtypes included diffuse large B-cell lymphoma in three (3.3\%), mantle cell lymphoma in one (1.1\%), and follicular lymphoma in one $(1.1 \%)$, respectively. Among 90 MZBCL patients, 79 formed the basis of the current analysis after excluding eleven. The reasons for exclusion were systemic involvement of MZBCL recognized thorough the staging work-up in two patients, previous or current history of malignancy other than MZBCL in four, and local recurrence following local excision alone or systemic chemotherapy alone (without RT) at other hospitals in five, respectively (Fig. 1).

\section{Radiation therapy}

RT was delivered to 121 eyes in 79 patients. All patients were placed in the supine position with thermoplastic mask for immobilization. A customized acryl contact lens, which was holding the lens shielding block to protect from radiation damage, was used individually (Fig. 2). After positioning this lens-shieling block in the eye, a single anterior oblique 6 or $9 \mathrm{MeV}$ electron beam was applied and the beam angle was determined according to the individual patient. The radiation dose was prescribed at $90 \%$ isodose line to adequately cover the anterior orbit. A total of 26 Gy was delivered with 2 Gy per fraction and five fractions per week.

\section{Follow-up and statistical analysis}

The initial response evaluation to RT was done in one to two months of RT completion by the responsible ophthalmologist. Complete remission (CR) was defined as complete resolution of clinical evidence of lymphoma in the conjunc- tival region by physical examination without orbital involvement. A reduction of at least $50 \%$ in tumor size was denoted as partial response (PR), whereas failure to attain the criteria was referred to as stable disease. Further follow-up evaluations were scheduled regularly by the responsible ophthalmologist, radiation oncologist, and / or medical oncologist at 6 months' interval thereafter. Physical examination, laboratory tests including $\mathrm{CBC}$ and LDH levels, and chest X-ray were performed on a regular basis, and imaging studies including $\mathrm{CT}$, magnetic resonance imaging, positron emission tomography-CT, esophagogastroduodenoscopy, or ultrasonography were optionally added. All relapsed cases were pathologically confirmed with biopsy. Late adverse events were also evaluated according to Common Terminology Criteria for Adverse Events ver. 4.0.

The durations of survival were from the last day of RT to the date of the first observed event or death, and the survival rates were calculated by Kaplan-Meier analysis. A p-value of less than 0.05 was considered statistically significant. Statistical analysis was performed with SPSS ver. 23.0 (IBM Corp., Armonk, NY) and SAS software ver. 9.4 (SAS Institute Inc., Cary, NC).

\section{Results}

\section{Patient characteristics}

The median age of all 79 patients was 38 years (range, 16 to 68 years) and about four-fifths were female (62 patients, 
A

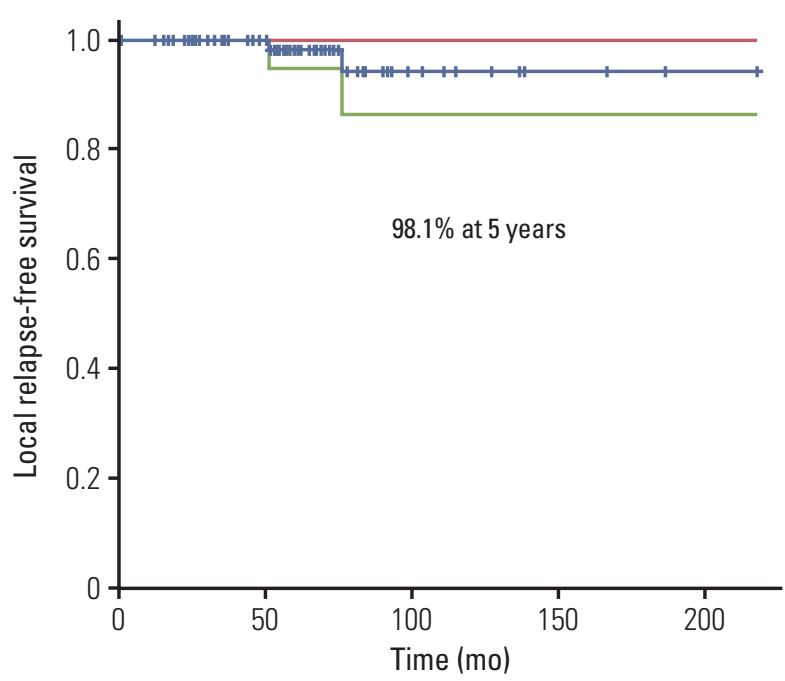

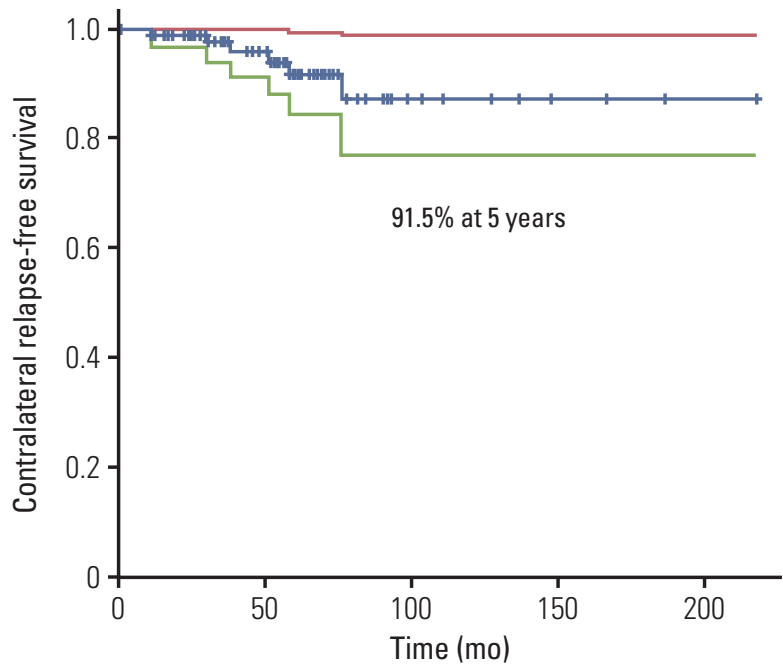

Fig. 3. Local relapse-free survival (A) and contralateral relapse-free survival (B) of 79 patients with primary conjunctival marginal zone B-cell lymphoma treated with radiation therapy.

78.5\%). About half of the patients (42 patients, 53.2\%) had synchronous bilateral involvement at time of diagnosis.

\section{Initial response, relapse patterns, and survival outcomes}

The initial response rate (CR+PR) following RT was 100\%: CR in 73 patients (92.4\%); and PR in six (7.6\%), respectively. All six patients who initially achieved PR exhibited gradual regression during the subsequent follow-up for 4-12 months. The median follow-up period of all the patients was 61.3 months (range, 5 to 149 months). Local recurrence within the radiation volume occurred in two patients $(2.5 \%)$ : one with unilateral involvement developed local relapse in 7 years and 1 month; and the other with bilateral involvement did bilateral relapse in 6 years and 4 months. These two patients, following re-staging work-up procedures, received re-RT with the same technique and were successfully salvaged until 5 years and 11 months and 4 years and 8 months following re-RT. Five patients $(6.3 \%)$ developed relapse at the initially uninvolved contralateral conjunctiva in the median 52.9 months following RT (range, 11 to 76 months). Among them, four patients were treated with re-RT and one declined re-RT and has been on regular monitoring. All five patients eventually achieved complete regression of recurrent lesion without recurrence. There was no patient who developed systemic relapse throughout the entire follow-up period. The 5 -year rates of overall survival, local relapse-free survival, and contralateral relapse-free survival were $100 \%, 98.1 \%$, and 91.5\%, respectively (Fig. 3). The initial response was not
Table 1. Detail of adverse events following radiation therapy

\begin{tabular}{lcccc} 
& Grade 1 & Grade 2 & Grade 3 & Total \\
\hline Dry eye & 16 & 5 & 0 & $21(26.6)$ \\
Eye pain & 3 & 1 & 0 & $4(5.1)$ \\
Tearing & 3 & 1 & 1 & $5(6.3)$ \\
Cataract & 4 & 0 & 1 & $5(6.3)$ \\
\hline
\end{tabular}

Values are presented as number $(\%)$.

associated with the risk of contralateral relapse ( $\mathrm{p}=0.51$ ). During the follow-up period, one patient was diagnosed as having pancreatic neuroendocrine tumor for which the patient underwent surgery.

\section{Adverse events}

The adverse events following RT are summarized in Table 1. Thirty-one patients among 79 (39.2\%) developed at least one of the adverse events related to RT: dry eye in $21(26.6 \%)$, eye pain in four $(5.1 \%)$, tearing in five $(6.3 \%)$, and cataract in five $(6.3 \%)$, respectively. Grade 3 adverse event was observed in only one patient $(1.3 \%)$ who developed tearing and cataract. 


\section{Discussion}

Through this retrospective study for a large series of conjunctival MZBCL patients from a single institute, we could depict clinical features and long-term outcomes of localized conjunctival MZBCL. In this series, the high rate of MZBCL in the conjunctival lymphoma $(102 / 107,95 \%)$ is a distinctive feature considering the result of previous studies on Western patients $(68.4,81.0 \%)$ [1,2]. The proportion of follicular lymphoma histology $(1 / 107,0.9 \%)$ also was much lower when compared with the previous Western studies $(8.0 \%-23 \%)$ $[1,2,17]$.

This patient series also denotes characteristic findings in demographic aspects. Age at diagnosis is younger than other ethnic groups; the mean age of Korean patients developing orbital or conjunctival MZBCL was in the forties [12], while that of other ethnic groups were in the sixties $[1,2,17,18]$. Female predominance of $78.5 \%$ is also a distinctive feature in the current study. Sex distribution in conjunctival lymphoma varies to the studies; meanwhile, Kirkegaard et al. [1] reported that there was no noticeable sex predilection in conjunctival MZBCL in the large-series multicenter study for Western patients $(1.05: 1)$ [2,19]. The high rate of bilateral involvement (53.2\%) is also a noticeable feature in this study compared with other studies (14.0\%-37.0\%) [1,2,10,15]. These demographic characteristics may suggest genetic and environmental influences on the pathogenesis of conjunctival MZBCL in Korean patients.

The standard RT dose has not been established yet in conjunctival MZBCL. Several studies were performed for ocular adnexal MZBCL to find optimal radiation dose schedule to minimize the risk of side effects $[1,7,9,12-15,20]$. Uno et al. [8] reported that radiation target volume should include the entire conjunctiva and the eyelid with the dose of $36 \mathrm{~Gy}$ in order to reduce the recurrence rate. On the other hand, radiation dose of 36 Gy was found to be accompanied by the adverse effects such as radiation-related retinopathy or corneal ulceration in many cases [8]. Bayraktar et al. [19] recommended that radiation dose of $\geq 30.6$ Gy be given to stage I ocular adnexal MZBCL to avoid recurrences. Woolf et al. [13] noted that $100 \%$ local control rates and acceptable toxicity could be achieved with the median dose of $30 \mathrm{~Gy}$ for the ocular adnexal lymphoma. International Lymphoma Radiation Oncology Group guideline demonstrated that the dose range is normally 20-30 Gy in indolent lymphoma including follicular lymphoma and marginal zone lymphoma and the lower end of this dose range is particularly in the eye because of deleterious ophthalmologic toxicity above 36 Gy [21]. FORT trial showed 24 Gy in 12 fractions is the effective RT regimen in the curative setting for indolent lymphoma, although $93 \%$ of patients were follicular lymphoma and $11 \%$ of patients were marginal zone lymphoma. Some authors showed 24-25 Gy of RT is effective for ocular MZBCL [18,22]. Tran et al. [18] showed excellent local control rate ( $100 \%$ at 2 years and $92 \%$ at 5 years) with the radiation dose as low as 24-25 Gy in 24 patients with ocular adnexal MZBCL. In the current study, excellent local control (98.1\% at 5 years) was achieved after 26 Gy of RT. A lower dose than 24 Gy might be sufficient in conjunctival MZBCL. Further clinical study is warranted to resolve this issue.

In the current study, local relapse was very low (2/79, $2.5 \%$ ), but contralateral relapse in initially uninvolved site was developed in five patients $(6.3 \%)$ after median 52.9 months following RT. This might be a progression from unilateral disease to bilateral disease or a preexisting subclinical microscopic disease which was overlooked at the initial evaluation $[20,23]$. The time to relapse following initial treatment for conjunctival MZBCL showed a wide range of 2-7 years in literature $[8,10,12,14]$, which was in concordance with our result of median 4.4 years. Accordingly, long-term follow-up would be necessary to detect local or contralateral relapse [15].

The lens of the eye is recognized as one of the most radiosensitive organ. The threshold for cataract formation after fractionated RT is known to be 4-5 Gy and about 15 Gy causes a 50\% probability of visual impairment [24]. Ohga et al. [20] reported the incidence of grade 2 or higher cataract to be $35.6 \%$ following median 30 Gy of RT for the ocular adnexa lymphoma. Harada et al. [15] also showed that cataracts developed in 36 of the 65 eyes $(55.4 \%)$ treated without lens shielding after median 30 Gy of RT for ocular adnexal MZBCL. The lens-shielding could be considered in the RT planning of conjunctival lymphoma otherwise the tumor is located very close to the lens. The development of cataract was reported from $9 \%[13,20]$ to $27.4 \%$ [15] with the lens shielding technique at $30 \mathrm{~Gy}$ of radiation for the ocular adnexa lymphomas. Uno et al. [8] reported that no cataract developed among 11 ocular adnexa lymphoma patients with lens shielding effort. In the current study, cataract developed in five patients $(6.3 \%)$. Our customized lens-shielding technique was very effective.

The current study has a limitation of retrospective study and selection bias from a single tertiary center. The predominance of MZBCL in conjunctiva and clinical features should be warranted in population-based study. On other hand, the current study has a few strong points: the largest number of patients with long-term follow-up was included; homogeneous staging work-up procedures were performed; and the standardized therapeutic protocol of RT with lens shielding was applied to all patients.

In conclusion, we observed younger age, female predominance and higher proportion of bilateral involvement in Korean conjunctival MZBCL patients. The 26 Gy of RT with 
lens-shielding technique was effective in achieving excellent local control and protecting from cataract formation. Longterm close monitoring would be necessary to detect local and contralateral conjunctival relapse.

\section{Conflicts of Interest}

Conflict of interest relevant to this article was not reported.

\section{References}

1. Kirkegaard MM, Coupland SE, Prause JU, Heegaard S. Malignant lymphoma of the conjunctiva. Surv Ophthalmol. 2015;60: 444-58.

2. Kirkegaard MM, Rasmussen PK, Coupland SE, Esmaeli B, Finger PT, Graue GF, et al. Conjunctival lymphoma: an international multicenter retrospective study. JAMA Ophthalmol. 2016;134:406-14.

3. Matsuo T, Ichimura K, Yoshino T. Spontaneous regression of bilateral conjunctival extranodal marginal zone B-cell lymphoma of mucosa-associated lymphoid tissue. J Clin Exp Hematop. 2007;47:79-81.

4. Tanimoto K, Kaneko A, Suzuki S, Sekiguchi N, Maruyama D, Kim SW, et al. Long-term follow-up results of no initial therapy for ocular adnexal MALT lymphoma. Ann Oncol. 2006;17: 135-40.

5. Han JJ, Kim TM, Jeon YK, Kim MK, Khwarg SI, Kim CW, et al. Long-term outcomes of first-line treatment with doxycycline in patients with previously untreated ocular adnexal marginal zone B cell lymphoma. Ann Hematol. 2015;94: 575-81.

6. Ferreri AJ, Govi S, Pasini E, Mappa S, Bertoni F, Zaja F, et al. Chlamydophila psittaci eradication with doxycycline as firstline targeted therapy for ocular adnexae lymphoma: final results of an international phase II trial. J Clin Oncol. 2012;30: 2988-94.

7. Ohga S, Nakamura K, Shioyama Y, Sasaki T, Yoshitake T, Atsumi K, et al. Radiotherapy for early-stage primary ocular adnexal mucosa-associated lymphoid tissue lymphoma. Anticancer Res. 2013;33:5575-8.

8. Uno T, Isobe K, Shikama N, Nishikawa A, Oguchi M, Ueno N, et al. Radiotherapy for extranodal, marginal zone, B-cell lymphoma of mucosa-associated lymphoid tissue originating in the ocular adnexa: a multiinstitutional, retrospective review of 50 patients. Cancer. 2003;98:865-71.

9. Ferrufino-Ponce ZK, Henderson BA. Radiotherapy and cataract formation. Semin Ophthalmol. 2006;21:171-80.

10. Hashimoto N, Sasaki R, Nishimura H, Yoshida K, Miyawaki D, Nakayama M, et al. Long-term outcome and patterns of failure in primary ocular adnexal mucosa-associated lymphoid tissue lymphoma treated with radiotherapy. Int J Radiat Oncol Biol Phys. 2012;82:1509-14.

11. Hoskin PJ, Kirkwood AA, Popova B, Smith P, Robinson M, Gallop-Evans E, et al. 4 Gy versus 24 Gy radiotherapy for patients with indolent lymphoma (FORT): a randomised phase 3 non-inferiority trial. Lancet Oncol. 2014;15:457-63.
12. Nam H, Ahn YC, Kim YD, Ko Y, Kim WS. Prognostic significance of anatomic subsites: results of radiation therapy for 66 patients with localized orbital marginal zone B cell lymphoma. Radiother Oncol. 2009;90:236-41.

13. Woolf DK, Kuhan H, Shoffren O, Akinnawo EM, Sivagurunathan $\mathrm{B}$, Boyce $\mathrm{H}$, et al. Outcomes of primary lymphoma of the ocular adnexa (orbital lymphoma) treated with radiotherapy. Clin Oncol (R Coll Radiol). 2015;27:153-9.

14. Goda JS, Le LW, Lapperriere NJ, Millar BA, Payne D, Gospodarowicz MK, et al. Localized orbital mucosa-associated lymphoma tissue lymphoma managed with primary radiation therapy: efficacy and toxicity. Int J Radiat Oncol Biol Phys. 2011;81:e659-66.

15. Harada K, Murakami N, Kitaguchi M, Sekii S, Takahashi K, Yoshio K, et al. Localized ocular adnexal mucosa-associated lymphoid tissue lymphoma treated with radiation therapy: a long-term outcome in 86 patients with 104 treated eyes. Int J Radiat Oncol Biol Phys. 2014;88:650-4.

16. Parikh RR, Moskowitz BK, Maher E, Della Rocca D, Della Rocca R, Culliney B, et al. Long-term outcomes and patterns of failure in orbital lymphoma treated with primary radiotherapy. Leuk Lymphoma. 2015;56:1266-70.

17. Ferry JA, Fung CY, Zukerberg L, Lucarelli MJ, Hasserjian RP, Preffer FI, et al. Lymphoma of the ocular adnexa: a study of 353 cases. Am J Surg Pathol. 2007;31:170-84.

18. Tran KH, Campbell BA, Fua T, MacManus M, Ryan G, Chesson B, et al. Efficacy of low dose radiotherapy for primary orbital marginal zone lymphoma. Leuk Lymphoma. 2013;54: 491-6.

19. Bayraktar S, Bayraktar UD, Stefanovic A, Lossos IS. Primary ocular adnexal mucosa-associated lymphoid tissue lymphoma (MALT): single institution experience in a large cohort of patients. Br J Haematol. 2011;152:72-80.

20. Ohga S, Nakamura K, Shioyama Y, Sasaki T, Yamaguchi T, Yoshitake T, et al. Treatment outcome of radiotherapy for localized primary ocular adnexal MALT lymphoma: prognostic effect of the AJCC tumor-node-metastasis clinical staging system. Anticancer Res. 2015;35:3591-7.

21. Yahalom J, Illidge T, Specht L, Hoppe RT, Li YX, Tsang R, et al. Modern radiation therapy for extranodal lymphomas: field and dose guidelines from the International Lymphoma Radiation Oncology Group. Int J Radiat Oncol Biol Phys. 2015;92: 11-31.

22. Goda JS, Gospodarowicz M, Pintilie M, Wells W, Hodgson DC, Sun A, et al. Long-term outcome in localized extranodal 
mucosa-associated lymphoid tissue lymphomas treated with radiotherapy. Cancer. 2010;116:3815-24.

23. Aronow ME, Portell CA, Rybicki LA, Sweetenham JW, Singh AD. Ocular adnexal lymphoma: assessment of a tumor-node- metastasis staging system. Ophthalmology. 2013;120:1915-9.

24. Henk JM, Whitelocke RA, Warrington AP, Bessell EM. Radiation dose to the lens and cataract formation. Int J Radiat Oncol Biol Phys. 1993;25:815-20. 